\title{
Multiple Cardiac Rhabdomyomas Visualised Using Micro-CT in a Case of Tuberous Sclerosis
}

\begin{abstract}
Cardiac rhabdomyoma is the most common tumour of the heart in infancy and childhood, representing approximately $60 \%$ of all primary cardiac tumours in these age groups. Though they have a tendency to regress with advancing age and are histologically benign, rhabdomyomas may cause mechanical obstruction to blood flow, arrhythmia, conges- tive cardiac failure and death and may be associated with underlying genetic syndromes such as tuberous sclerosis. We present the case of a primigravida in her early 20 s with no significant medical history who was referred to the Fetal Medicine Unit at 34 weeks' gestation following the detection of an irregular fetal heartbeat. An anomaly scan at 20 weeks
\end{abstract}

Introduction Antenatal Sonography at $\mathbf{3 4}+\mathbf{5}$ weeks demonstrated the presence of several intracardiac lesions, suspicious of rhabdomyomas. Confirmation of the diagnosis was re- quested from using the less invasive autopsy service at our institution. Post-mortem whole-body CT and MRI demonstrated cortical tubers and subependymal nodules, but no rhabdomyomas were identified. The heart was removed at limited autopsy and imaged using micro-CT. This revealed over 20 clearly visualised individual rhab- domyomas, widely dispersed throughout the heart. We present the first description of cardiac tumour identifica- tion using micro-CT technology.

\section{Case Presentation}

A primigravida mother with no significant medical history presented to a Fetal Medicine Unit at 34weeks' gestation follow- ing the detection of an irregular fetal heartbeat by the Commu- nity Midwife. An anomaly scan undertaken at 20 weeks had been reported as normal. Antenatal ultrasound at $34+5$ weeks demonstrated the presence of several intracardiac lesions, suspicious of rhabdomyomas. The parents opted to terminate the pregnancy. Post-mortem CT and MRI demonstrated intracranial tubers and subependymal nodules, but no rhabdomyomas. The heart was re- moved at autopsy and imaged using micro-CT (fig. 1), which demonstrated multiple individual rhabdomyomas, widely dis- persed throughout the heart, in keeping with tuberous sclerosis (fig. 2). To our knowledge, this is the first description of human fetal cardiac tumour imaging using micro-CT technology. Micro- CT techniques are traditionally associated with non-destructive testing in the industry, but are gaining traction in biomedical re- search [1].

Cardiac rhabdomyoma is the most common tumour of the heart in infancy and childhood, representing approximately $60 \%$ of all primary cardiac tumours in these age groups [2]. Though they have a tendency to regress with advancing age and are histo- logically benign, rhabdomyomas may cause mechanical obstruc- tion to blood flow, arrhythmia, congestive cardiac failure and death. It is estimated that the incidence of tuberous sclerosis in patients with cardiac rhabdomyoma is $60-80 \%$ [3]. 